\title{
THE LOWER CENTRAL SERIES IN SOME GROUPS WITH THE SUBNORMAL JOIN PROPERTY
}

\author{
HOWARD SMITH ${ }^{1}$
}

\begin{abstract}
The following question is considered: What groups $G$ are such that, given any subnormal subgroups $H$ and $K$ of $G$, with join $J$, and given any positive integers $a$ and $b$, there exists an integer $c$ such that $\gamma_{c}(J)$ is contained in $\gamma_{a}(H) \gamma_{b}(K)$ ? It is shown that many, but not all, groups known to have the "subnormal join property" satisfy this further condition.
\end{abstract}

Suppose the group $G$ is generated by subnormal subgroups $H$ and $K$, and let $a, b$ be given positive integers. It was proved in [1, Theorem $\mathrm{A}]$ that there is an integer $c$ such that $\gamma_{c}(G) \leqslant \gamma_{a}(H) \gamma_{b}(K)$, provided that $G / G^{\prime}$ is finitely generated. (In [2, Theorem B] it was shown that $G / G^{\prime}$ need only have finite rank.) Further results of this kind appear in [11]: for instance, the case where the tensor product of $H / H^{\prime}$ and $K / K^{\prime}$ has finite rank modulo its divisible torsion radical. Another sufficient condition is when $G=H K$, that is, when $H$ and $K$ permute [10, Lemma 2].

The question discussed here is this: What groups $G$ are such that, whenever $H$ and $K$ are subnormal subgroups of $G$ and $a, b$ are given integers, there is an integer $c$ such that $\gamma_{c}(\langle H, K\rangle) \leqslant \gamma_{a}(H) \gamma_{b}(K)$ ? Denoting the class of such groups by $\mathfrak{Z}$, perhaps the first observation to make is that $\mathfrak{L}$ is closed under the operations of forming normal subgroups and homomorphic images (that is, $\mathfrak{R}=\operatorname{sn} \mathfrak{l}=Q \mathfrak{R}$ ). Further, in [9] we considered a related class of groups denoted there by $\mathfrak{X}_{1}$ and characterized by the property that it is the largest $\langle$ sn, $Q\rangle$-closed class $\mathfrak{X}$ such that the join of any two subnormal nilpotent subgroups of an $\mathfrak{X}$-group is again nilpotent. Since it was also shown [9, Theorem 4.3] that $\mathfrak{X}_{1}$ is contained in $\Im$, the class of groups with the "subnormal join property", we immediately have that $\mathfrak{\Omega} \subseteq \mathfrak{X}_{1} \subseteq \subseteq$. At present, no example is known of an $\subseteq$-group which is not in $\mathfrak{X}_{1}$, but it is not difficult to distinguish between $\mathfrak{Z}$ and $\mathfrak{X}_{1}$ :

THEOREM 1. There is a group $G$ not in the class 2 which has the property that the join of any two subnormal nilpotent subgroups of $G$ is again subnormal and nilpotent.

Proof. Let $p$ be a prime, $A$ an infinite elementary abelian $p$-group, and $B$ a group of order $p$. Define $H$ to be the restricted wreath product of $B$ by $A$, let $C$ be another

Received by the editors April 25, 1983.

1980 Mathematics Subject Classification. Primary 20E15, 20 F14.

${ }^{1}$ The author gratefully acknowledges support from both the University of Wales (Faculty of Science Bursary) and the Department of Mathematics at York University. 
$p$-cycle, and set $G=H \operatorname{wr} C$. Clearly $G$ is soluble and has exponent $p^{3}$, and so $G$ is a Baer group (see [5, Theorem 7.17]). In particular, $C$ is subnormal in $G$. Now $H \triangleleft^{2}$ $G=\langle H, C\rangle$, and so, if $G \in \mathfrak{L}$, we would have $\gamma_{c}(G) \leqslant H$ for some integer $c$, and, hence, $\gamma_{c}\left(H^{x}\right) \leqslant H$ for any element $x$ of $C$, which would imply that $H$ is nilpotent. Since $H$ has trivial centre, we conclude that $G \notin \mathfrak{R}$. However, from [9, Theorems 4.7 and 4.9], we know that $G \in \mathfrak{X}_{1}$ (since $G$ is metabelian-by-finite).

With the notation of Theorem 1, we note that if $D$ is a subgroup of $H$ such that $D C=C D$, then $D=1$; that is, the permutizer of $C$ in $H$ is trivial. Since $H$ is not nilpotent, there is certainly no integer $n$ such that $\gamma_{n}(H)=1$. This supplies an answer to a question posed in [7].

The following "reduction lemma" will be of use in determining which groups belong to $\mathfrak{R}$. This reduction appears in the proof of Theorem A of [1] (except that no reference to the class $\subseteq$ is necessary there) and so the proof is omitted.

Lemma 1. Suppose $\mathfrak{X}=\operatorname{sn} \mathfrak{X}=Q \mathfrak{X}$ is a subclass of $\mathfrak{S}$. Then $\mathfrak{X} \subseteq \mathfrak{L}$ if and only if, given an abelian-by-nilpotent group $G$ in the class $\mathfrak{X}$, where $G=\langle H, K\rangle$ and $H, K$ are subnormal in $G$, and given any positive integers $a$ and $b$, there exists an integer $c$ such that $\gamma_{c}(G) \leqslant \gamma_{a}(H) \gamma_{b}(K)$.

We know from [3, Corollary 1 to Lemma 2.3] that if the group $G$ has a nilpotent derived subgroup, then $G$ belongs to $\subseteq$. Such groups may easily be shown to lie in $\mathfrak{L}$ without recourse to the above lemma. Assuming only that $G=\langle H, K\rangle$, where $H, K$ are subnormal, and noting that $[H, K]$ is nilpotent, two successive applications of [10, Lemma 2] to the groups $H^{K}=H[H, K]$ and $G=H^{K} \cdot K$ give us the required result. Thus we have

THEOREM 2. Nilpotent-by-abelian groups belong to the class $\mathfrak{2}$.

As an example of the use of Lemma 1, we shall prove the following (which may, however, be stated as a corollary of Theorem 4).

THEOREM 3. If $G$ is a group such that $G^{\prime}$ satisfies max-sn (the maximal condition for subnormal subgroups), then $G \in \mathfrak{R}$.

Proof. Let $G$ be as stated. Then $G \in \mathbb{S}[3$, Theorem 4.3(i)] and hence, by Lemma 1 , we may assume that there is an integer $r$, such that $\gamma_{r}(G)=A$ is abelian, and $G=\langle H, K\rangle$, where $H$ and $K$ are subnormal in $G$. Now $G^{\prime}$ is soluble and therefore polycyclic. In particular, $G^{\prime}$ is finitely generated (f.g.) and is thus contained in a f.g. subgroup of $G$ of the form $G_{0}=\left\langle H_{0}, K_{0}\right\rangle$, where $H_{0} \leqslant H$ and $K_{0} \leqslant K$. Let integers $a, b$ be given. Without loss of generality we take $a, b \geqslant r$, so that $\gamma_{a}(H)$ and $\gamma_{b}(K)$ permute. Suppose we have found an integer $d$ such that $\gamma_{d}\left(G^{\prime}\right) \leqslant \gamma_{a}(H) \gamma_{b}(K)$. Then the proof is again completed by application of [10, Lemma 2] to $K G^{\prime}$ and $G$, respectively. Since $A \leqslant G^{\prime}$, we have $G_{0}=\left\langle H_{0} A, K_{0} A\right\rangle$, a join of two f.g. subnormal subgroups (since $G / A$ is nilpotent). Let $u, v$ be such that $\gamma_{u}(H A) \leqslant \gamma_{a}(H), \gamma_{v}(K A)$ $\leqslant \gamma_{b}(K)$. Then, by [1, Theorem A], there is an integer $d$ such that $\gamma_{d}\left(G_{0}\right) \leqslant$ $\gamma_{u}\left(H_{0} A\right) \gamma_{v}\left(K_{0} A\right) \leqslant \gamma_{a}(H) \gamma_{b}(K)$. But $G^{\prime} \leqslant G_{0}$ and so the theorem is proved. 
Now the example given in Theorem 1, when considered in the light of Theorem 2 , shows that an extension of an $\mathfrak{R}$-group by a finite group need not belong to $\mathfrak{R}$. Thus

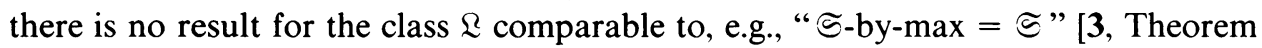
5.1] or " $\mathfrak{X}_{1}$-by-max $=\mathfrak{X}_{1}$ " (a consequence of [9, Theorem 4.7(i)]). The question as to whether, in the reverse direction, it is true that max-by- $\mathfrak{R}=\mathfrak{R}$, or even that finite-by- $\mathfrak{R}=\mathfrak{R}$, is left open here. (Similar results do in fact obtain for both $\subseteq$ and $\mathfrak{X}_{1}$-see [4, Theorem 6.2 and 9, Theorems 2.2 and 4.6(i)].)

The obvious approach is then to consider classes of groups known to be contained in $\mathfrak{X}_{1}$ (but described of course in terms of classes other than $\mathfrak{X}_{1}$ ) and to try to determine whether they lie in $\mathfrak{L}$. Let $\mathfrak{A}, \mathfrak{B}, \mathfrak{F}_{r}$ denote, respectively, the classes of abelian groups, groups with bounded subnormality indices, and groups having finite (Prüfer) rank. Then an examination of the results given in $\$ 4$ of [9] reveals that the most extensive class of soluble groups known to lie in $\mathfrak{X}_{1}$ and worth considering here (in view of Theorems 1 and 2 above) is, in the usual notation of class products, $\mathfrak{F}_{r}\left(\mathfrak{B}\left(\mathfrak{F}_{r} \mathfrak{U}\right)\right) \cap P(\mathfrak{U})$. We shall in fact prove

THEOREM 4. $\mathfrak{F}_{r}\left(\mathfrak{B}\left(\mathfrak{F}_{r} \mathfrak{A}\right)\right) \subseteq \mathfrak{R}$.

It is evident from Lemma 1 that $\mathfrak{F}_{r}$ may be replaced, in either of the two places in which it appears in the statement of the above theorem, by any $\langle$ sn, $Q\rangle$-closed class $\mathfrak{X}$ of groups with the property that soluble $\mathfrak{X}$-groups have finite rank. (It should be mentioned that this reduction to the soluble case depends only on Lemma 5 of [7].) This situation is similar to those described in [9], and we need not list the possibilities for $\mathfrak{X}$ here. The main requirements for the proof of Theorem 4 are, of course, the results from [1, 2, and 10] mentioned above, together with the corresponding results (from [1 and 2]) on integral augmentation ideals. As a minor remark, we note that Proposition $\mathrm{C}$ of [1] contains no reference to a bound for the integer obtained, but the required improvement is so easily deduced that we shall treat it as implicit in the statement of that result. The following lemma will also be required. Let $\mathfrak{B}_{i}$ denote the class of groups $G$ in which each subnormal subgroup has defect at most $i$. Then, as an easy consequence of Theorem 1 of [6] we have

LEMMA 2. If the group $G$ belongs to the class $\mathfrak{B}_{i}$, then there is an integer $f=f(i)$ such that $\gamma_{f+1}(G)=\gamma_{f}(G)$.

Proof of Theorem 4. Let $G$ be a group, and suppose $M, N$ are normal subgroups of $G$ such that (i) $M \leqslant N \leqslant G^{\prime}$, (ii) $M, G^{\prime} / N$ have finite ranks $r, s$, respectively, and (iii) $N / M \in \mathfrak{B}_{i}$. Let $a_{1}, b_{1}$ be given integers. We require an integer $c$ such that $\gamma_{c}(\langle H, K\rangle) \leqslant \gamma_{a_{1}}(H) \gamma_{b_{1}}(K)$, where $H, K$ are subnormal subgroups of $G$. By Lemma 1 we may suppose that $G=\langle H, K\rangle$ and that $\gamma_{n}(G)=A$ is abelian for some integer $n$. Further, by [10, Lemma 2], we may assume that $A \leqslant H \cap K$. It thus suffices to show that, for given $a, b$, there is an integer $d$ such that $\left[A,{ }_{d} G\right] \leqslant\left[A,{ }_{a} H\right]\left[A,{ }_{b} K\right]$.

Let $H_{0}, K_{0}$ be subgroups of $H, K$ such that $H_{0} / A, K_{0} / A$ are finitely generated, and write $G_{0}=\left\langle H_{0}, K_{0}\right\rangle$. Then, by an application of [1, Theorem B], there is an integer $d_{1}$ such that $\left[A,{ }_{d_{1}} G_{0}\right] \leqslant\left[A,{ }_{a} H_{0}\right]\left[A,{ }_{b} K_{0}\right]=B$, say. If we can find a suitably bounded $d$ such that $\left[A,{ }_{d} G_{0}\right] \leqslant B$, then the proof will, of course, be complete. Factoring by $\left[A,{ }_{d_{1}} G_{0}\right]$, we suppose that $G_{0}$ is nilpotent. 
Now write $N_{0}=G_{0} \cap N, M_{0}=G_{0} \cap M$. Then $N_{0} / M_{0}$ is a nilpotent group in the class $\mathfrak{B}_{i}$ (here we are using the fact that $G_{0}$ is subnormal in $G$ ), and, hence, by Lemma 2, the nilpotency class of $N_{0} / M_{0}$ is at most $f=f(i)$. Let $g=\max (n, f+1)$. Then $\gamma_{g}\left(N_{0}\right) \leqslant M_{0} \cap A$, and so we may assume that $M_{0} \leqslant A$. In considering the action of $G_{0}$ on $A$, it may further be assumed, as in the proof of the Proposition in [8], that $A$ is a finite $p$-group.

Let $C_{0}=C_{G_{0}}\left(M_{0}\right)$. Then $G_{0} / C_{0}$ may be viewed as a subgroup of Aut $M_{0}$. As a finite $p$-group, $G_{0} / C_{0}$ therefore has rank at most $t=\frac{1}{2} r(5 r-1)$ (a result due to P. Hall-see [5, Lemma 7.44]). Regarding $M_{0}$ as a $\mathbb{Z}\left(G_{0} / C_{0}\right)$-module, we may apply Theorem $\mathrm{C}$ of [2] to deduce that, for some integer $d_{2}=d_{2}(a, b, n, t)$,

$$
\left[M_{0},{ }_{d 2} G_{0}\right] \leqslant\left[M_{0},{ }_{a} H_{0}\right]\left[M_{0},{ }_{b} K_{0}\right] \leqslant B .
$$

Thus, writing $h=g+d_{2}$, we have $\left[A,{ }_{h} N_{0}\right] \leqslant B$.

Next, let $C=C_{G_{0}}(A)$. Then, modulo $C N_{0},\left[H_{0}, K_{0}\right]$ is a finite $p$-group of rank $s$ and is thus contained (again modulo $C N_{0}$ ) in a $2 s$-generator subgroup of the form $G_{1}=\left\langle H_{1}, K_{1}\right\rangle$, where $H_{1} \leqslant H_{0}$ and $K_{1} \leqslant K_{0}$ (here we use Burnside's Basis Theorem, as in the proof of Lemma 5 of [8]). Now we regard $A$ as a $\mathbb{Z}\left(G_{1} C / C\right)$-module and again apply [2, Theorem C] to obtain an integer $d_{3}=d_{3}(a, b, s, n)$ such that $\left[A,{ }_{d_{3}} G{ }_{1} C\right] \leqslant\left[A,{ }_{a} H_{1}\right]\left[A,{ }_{b} K_{1}\right] \leqslant B$.

Write $G_{2}=G_{1} C N_{0}$. Then, using [1, Proposition C], we deduce that $\left[A,{ }_{d_{4}} G_{2}\right] \leqslant B$, where $d_{4}=d_{4}\left(d_{3}, h, n\right)$. Finally, since $\left[H_{0}, K_{0}\right] \leqslant G_{2}$, and $G_{0}=H_{0} K_{0}\left[H_{0}, K_{0}\right]$, two further applications of Proposition $\mathrm{C}$ of [1] give the desired result.

\section{REFERENCES}

1. J. C. Lennox, D. Segal and S. E. Stonehewer, The lower central series of a join of subnormal subgroups, Math. Z. 154 (1977), 85-89.

2. J. C. Lennox and S. E. Stonehewer, The join of two subnormal subgroups, J. London Math. Soc. (2) 22 (1980), 460-466.

3. D. J. S. Robinson, Joins of subnormal subgroups, Illinois J. Math. 9 (1965), 144-168.

4. On the theory of subnormal subgroups, Math. Z. 89 (1965), 30-51.

5. Finiteness conditions and generalised soluble groups, Vol. 2, Springer-Verlag, Berlin and New York, 1972.

6. J. E. Roseblade, On groups in which every subgroup is subnormal, J. Algebra 2 (1965), 402-412.

7. $\quad$ The derived series of a join of subnormal subgroups, Math. Z. 117 (1970), 57-69.

8. H. Smith, Commutator subgroups of a join of subnormal subgroups, Arch. Math. (Basel) 41 (1983), 193-198.

9. Groups with the subnormal join property, Canad. J. Math. (to appear).

10. S. E. Stonehewer, Nilpotent residuals of subnormal subgroups, Math. Z. 139 (1974), 45-54.

11. J. P. Williams, The join of several subnormal subgroups, Proc. Cambridge Philos. Soc. 92 (1982), $391-399$.

Department of Mathematics, York University, Downsview, Ontario, Canada

Current address: Department of Mathematics, University College, Cardiff, Wales, United Kingdom 